\title{
Ferrimagnetic Resonance in Polycrystalline Ferrite and Garnet Disks at L-Band Frequencies
}

\author{
W. E. Case, R. D. Harrington, and L. B. Schmidt
}

(January 15, 1964)

\begin{abstract}
An experimental study made on the measurement of the gyromagnetic ratio and line width of four ferrimagnetic materials at L-band using thin disk-shaped samples in a rectangular $\mathrm{TE}_{102}$ mode cavity at $1107 \mathrm{Mc} / \mathrm{s}$ is described. The study emphasized ferrimagnetic resonant measurements on a one half inch diameter sample of a given material as a function of aspect ratio. Experimental results indicate that for three materials the gyromagnetic ratio could be accurately calculated from Kittel's equation for aspect ratios of 50 or greater. Because of an anomaly, the calculation was impossible for a fourth material, a nickel aluminum ferrite. Generally, very high aspect ratios are required to cause line width measurements to rapidly approach an asymptotic value.
\end{abstract}

\section{Introduction}

Considerable effort has recently been directed towards the development of ferrite and garnet devices such as isolators and circulators at the lower microwave frequencies. However, there is a lack of engineering information available on the line width and gyromagnetic ratio of ferrites at these frequencies even though these quantities are basic to the design of microwave devices. One of the simpler methods of measuring line width, $\Delta H$, and the gyromagnetic ratio, $\gamma$, at higher microwave frequencies such as X-band has been based on the determination of the absorption of a small spherical sample in a nondegenerate rectangular mode cavity $[1,2] .{ }^{1}$ This technique can be extended to L-band frequencies although in many cases it may be necessary to use thin polycrystalline disk-shaped samples rather than spheres in order to avoid magnetic saturation problems [3, 4]. However, in the case of disks, it is necessary to apply demagnetization corrections in calculating $\gamma$, as was pointed out by Kittel [5]. Furthermore, at L-band frequencies, it is necessary to use a disk orientation in the cavity which is more susceptible to dielectric effects than is usually the case at higher frequencies where smaller cavities of different modes may be used. It was thus the purpose of this study to determine the effects of sample size and geometry upon the accuracy of L-band ferrimagnetic magnetic resonance measurements. This involved an experimental study of line width and gyromagnetic ratio as a function of aspect ratio (diameter/thickness) of several ferrites.

\section{Experimental Techniques}

The development of a ferrimagnetic resonance measurement facility at L-band frequencies is subject to two basic requirements. First, the d-c field must be perpendicular to the plane of the diskshaped sample and the RF field in order to avoid low field losses [4]. Secondly, the cavity must be designed so that it can be placed between the pole caps of a typical electromagnet. This latter requirement becomes somewhat more severe at L-band due to the large size cavity that must be utilized. However, both requirements are readily satisfied by placing the disk-shaped sample at the center of a $\mathrm{TE}_{102}$ rectangular transmission cavity (fig. 1), as was pointed out by Sirvetz and Zneimer [3].

The measurement procedure utilized for obtaining' data in this study is similar to an attenuation technique which is being issued as an ASTM tentative method of test for Ferrimagnetic Resonance Line Width and Gyromagnetic Ratio of Nonmetallic

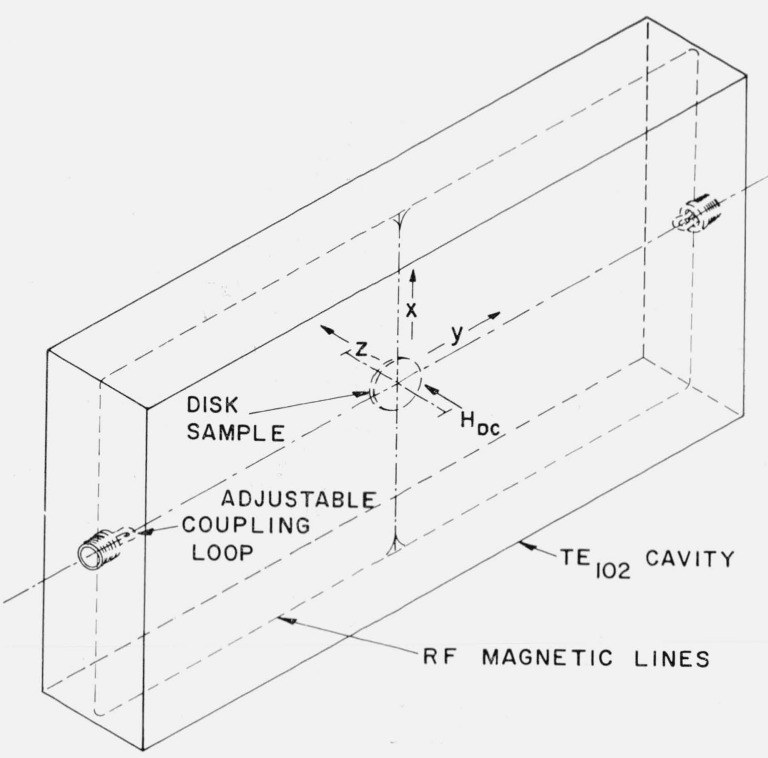

FIgURE 1. Rectangular transmission cavity using $T E_{102}$ mode at $1107 \mathrm{Mc} / \mathrm{s}$. 
Magnetic Materials designated ASTM C524-63T. Our setup varies from the ASTM method in that the input power to the cavity is held constant and measurements are made with a precision attenuator located in the IF detection circuit following the cavity. The frequency is adjusted to give cavity resonance for the empty cavity, and the variable precision IF attenuator is set to $A_{0}$ to give a convenient output reference level, as indicated on the output detector. The sample is then inserted, the d-c magnetic field adjusted for maximum absorption in the sample, the frequency adjusted for cavity resonance, and the precision attenuator set to obtain the same output reference signal. The new attenuator reading is defined as $A_{r}$, and the d-c field reading is defined as $H_{r}$. The attenuator reading corresponding to the half-power points on the ferrimagnetic resonance loss curve of the sample is calculated from the following formula [6]:

$$
A_{1 / 2}=A_{0}+20 \log 2-20 \log \left(10^{\frac{A_{0}-A_{r}}{20}}+1\right) .
$$

The attenuator is now set at the calculated $A_{1 / 2}$ value. The $d-c$ magnetic field necessary to return the output power to its previous level on one side of resonance is then read and recorded. The d-c field is then similarly adjusted and recorded for the other side of resonance. The difference between these two d-c field measurements is the line width, $\Delta H$.

The effective oyromagnetic ratio for the case of a sphere is determined from the formula

$$
\gamma=\frac{2 \pi f_{r}}{H_{r}}
$$

where $f_{r}$ is the microwave frequency required for cavity resonance at maximum sample absorption and $H_{r}$ is the corresponding d-c field. In the case of a disk magnetized perpendicular to its plane, the gyromagnetic ratio is given by

$$
\gamma=\frac{2 \pi f_{r}}{H_{r}-\left(N_{z}-N_{x}\right) M_{z}}
$$

which is Kittel's equation where the demagnetizing factors $N_{x}$ and $N_{y}$ are equal and $N_{x}+N_{y}+N_{z}=4 \pi$. $M_{z}$ is the magnetic moment per unit volume.

As can be seen in figure 1, the sample is oriented with its plane perpendicular to the $E$-field in the cavity. Even though it is at an electric field node, the diameter of the sample must be restricted in order to avoid errors due to dielectric effects. At higher frequencies where smaller cavities may be used, it is possible to pick a cavity mode in which the plane of a disk may be parallel to the electric field in the cavity. In these cases, dielectric effects are avoided by having a sufficiently thin sample located at an electric field node [7]. The necessity of placing the plane of the disk perpendicular to the $E$-field in the L-band cavity is not a serious problem due to the longer wavelength involved. This was experimentally verified by comparing $H_{r}$ and line width data on disks of $1 / 2$ in. diam with corresponding data on the same disk reduced to $1 / 4 \mathrm{in}$. diam and ground to the same aspect ratio. Resonant field measurements agreed within 0.3 percent for a given sample for three different materials. The dielectric effect on $H_{r}$ may thus be considered negligible. For a fourth material, a distinct diameter dependence anomaly was observed, as will be described below. Since the dielectric constant of this material is comparable to that of the other three materials, it is believed that this anomaly is not due to dielectric effects. In the case of line width measurements, the variation between different diameter disks may be as great as 5 percent for all four materials. This small diameter dependence was not considered a serious error since the accuracy of the line width measurements due to sample variations, etc., was also of the order of 5 percent. Furthermore, it will be noted later that much larger errors in line width may arise unless disks having large diameter to thickness ratios are used. It appears that a $1 / 2$ in. disk offers a good compromise for keeping dielectric effects on line widths low while attaining high aspect ratios.

The samples were supported by a rexolite holder at the center of the cavity. The dielectric constant of the rexolite introduced negligible error in the measurement, as was determined from similar measurements using a polyfoam holder.

Possible errors due to wall effect were checked by measuring the disk at various distances from the broad wall of the cavity. The sample is normally placed at $1 / 2$ of the distance between the broad walls. Placing the sample $1 / 4$ and $1 / 8$ of this distance from one of the walls resulted in only negligible change in the resonant measurements.

Many of the materials designed for use at the low microwave frequencies have relatively low Curie temperatures which may introduce errors in the data due to ambient temperature fluctuations. This situation is more serious in disk samples since the temperature dependent magnetization appears in the gyromagnetic ratio formula as given in eq (2). These effects were essentially a voided by stabilizing the cavity temperature by means of coils carrying constant temperature water.

Having experimentally demonstrated that the above effects do not introduce appreciable errors in the measurements, the majority of the effort was directed towards a study of the dependence of $\Delta H$ and $\gamma$ on the aspect ratio $\left(\frac{\text { diameter }}{\text { thickness }}\right)$ of the disks. Early measurements indicated that more consistent data could be obtained from a given sample that was alternately ground down and measured rather than comparing data from several different samples of the same material. Initially, ultrasonic machining and diamond wheel grinding were used to cut a disk sample to a given diameter and thickness. A small rotary grinder using aluminum oxide optical finishing powder was then used to grind off small increments of thickness allowing many measurements on the same sample with a given diameter. In general there 
appeared to be no dependence of the resonance properties on surface finish for three of the materials, as was determined by using powders of different grit size. However, in the material noted above which showed anomalous effects for $H_{r}$, a surface finish dependence was noted.

\section{Results}

The four commercially available materials referred to above and for which the line width, $\Delta H$, and resonant field, $H_{r}$, were determined as a function of the aspect ratio of the disks have the following general composition:

Sample A - Yttrium iron garnet

Sample B-Magnesium, manganese, aluminum ferrite

Sample C-Substituted yttrium iron garnet

Sample D - Nickel aluminum ferrite.

All measurements were made at $1107 \mathrm{Mc} / \mathrm{s}$.

\subsection{Gyromagnetic Ratio}

Figure 2 shows $H_{r}$ versus aspect ratio for the first three materials above. It can be seen that in at least two cases an asymptotic value of resonant field

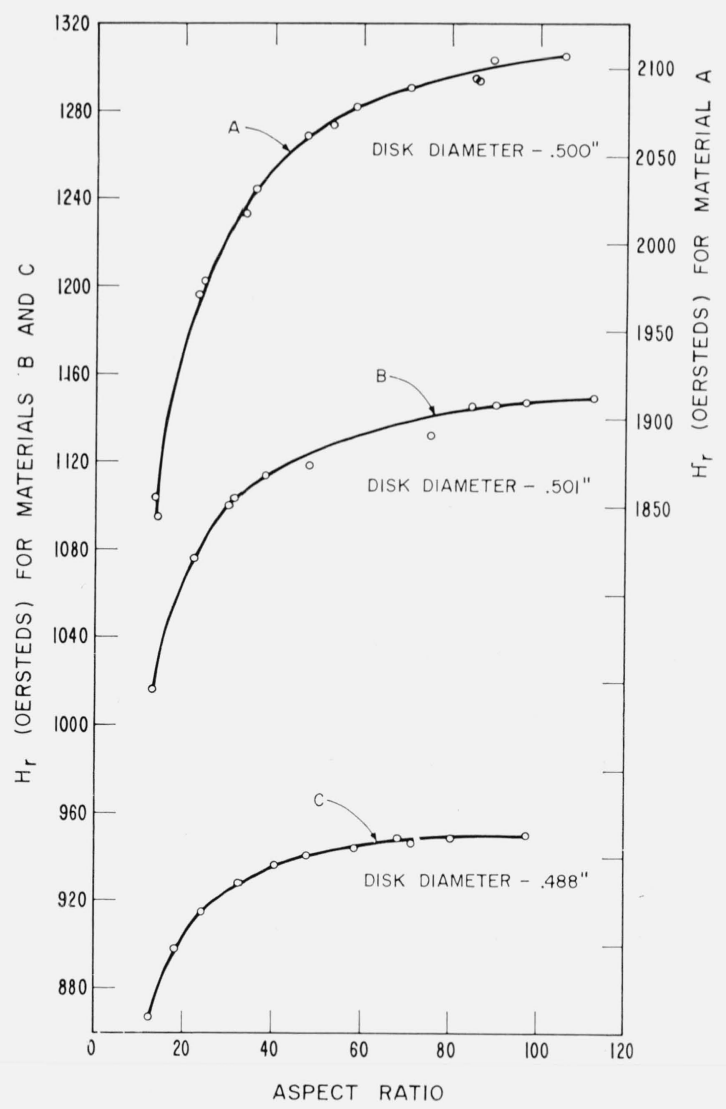

Figure 2. Resonant field, $H_{\mathrm{r}}$, versus aspect ratio for disk samples of three materials, $A, B$, and $C$. was not reached even for aspect ratios greater than 100 , which in this case corresponds to disks less than 0.005 in. in thickness. This of course indicates that in such measurements it is not, in general, reasonable to assume that one has an infinitely thin disk such that demagnetization corrections are not needed. However, these curves essentially express the dependence of Kittel's relation as given in (2) on demagnetizing factors which are a function of the aspect ratio. The relationship between the demagnetizing factors of ellipsoidal samples and their aspect ratios has been given by Stoner [8]. Thus $\left(N_{z}-N_{x}\right)$ for a given disk may be readily obtained if we assume that the demagnetizing factors of an ellipsoid and disk of the same aspect ratio are identical. The value of magnetization, $M_{z}$, may be determined from an independent measurement using a magnetometer. Thus, knowing $M_{z}$ and the value of $N_{z}-N_{x}$ as well as $H_{r}$ as given on the curve for a given aspect ratio, one can calculate the gyromagnetic ratio from eq (2). This should be a constant, independent of aspect ratio. When this was done for the data shown in figure 2, it was found that the variation in $\gamma$ as a function of aspect ratio for ratios of 50 or higher was less than 1 percent for each of the three samples. This was considered as satisfactory agreement between Kittel's equation and the observed data.

The validity of the assumption of equivalent demagnetizing factors for disks of the same aspect ratio was further substantiated by grinding ellipsoids from several disks keeping the aspect ratio approximately constant. A negligible effect on the measured value of $H_{\tau}$ was observed. This assumption thus appears to be valid at least for polycrystalline materials for measurements of this type. It has been noted in the literature that this situation may not necessarily be true for other types of measurements such as the study of magnetostatic modes in single crystal disks [9].

The above discussion implies that Kittel's equation is quite applicable for predicting the gyromagnetic ratio of most samples. However, similar measurements taken on Material D, which is a nickel aluminum ferrite, showed an anomalous behavior as can be seen in figure 3. This material varies from the previous ones described in that $H_{r}$ decreases for the higher aspect ratios and the data is strongly diameter dependent. Recent work indicates that $H_{r}$ is also dependent upon surface finish. The cause of this behavior is not yet understood. However, it is apparent that Kittel's equation as given in (2) is not applicable for describing this material. It thus appears that care should be taken in applying Kittel's equation to an unknown sample without first determining that the material is not following an unusual behavior. This implies that it may be necessary to measure samples of several different aspect ratios when gyromagnetic ratio data is desired. 


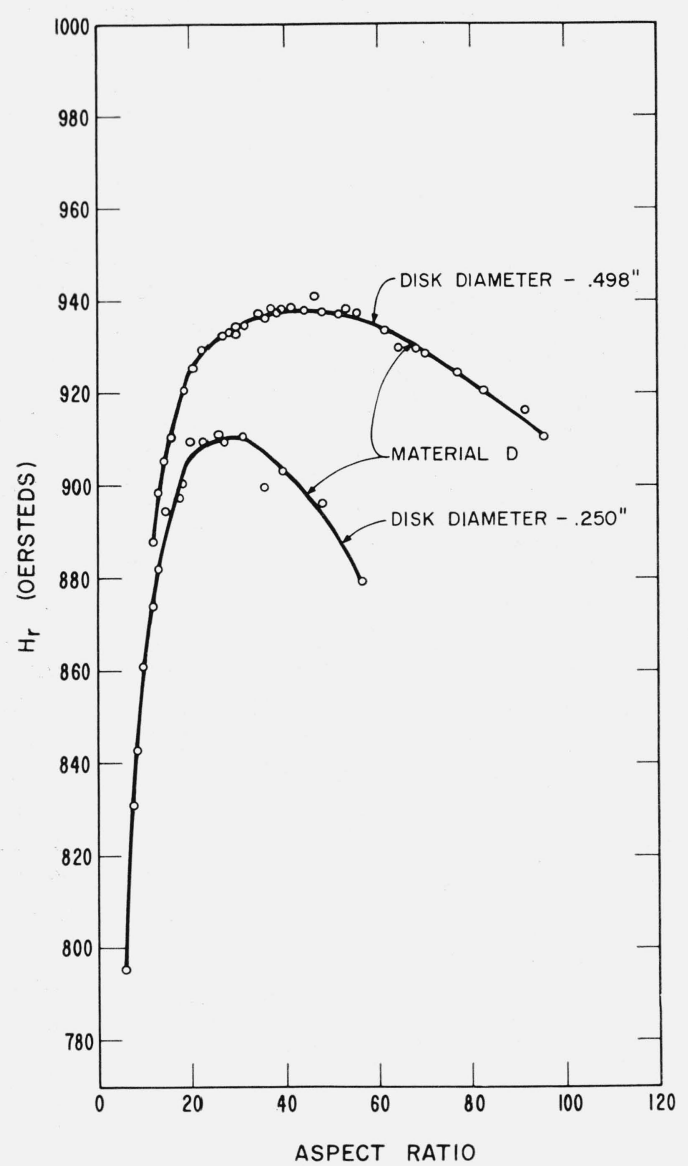

FiguRE 3. Resonant field, $H_{\mathrm{r}}$, versus aspect ratio for disk sample of material $D$.

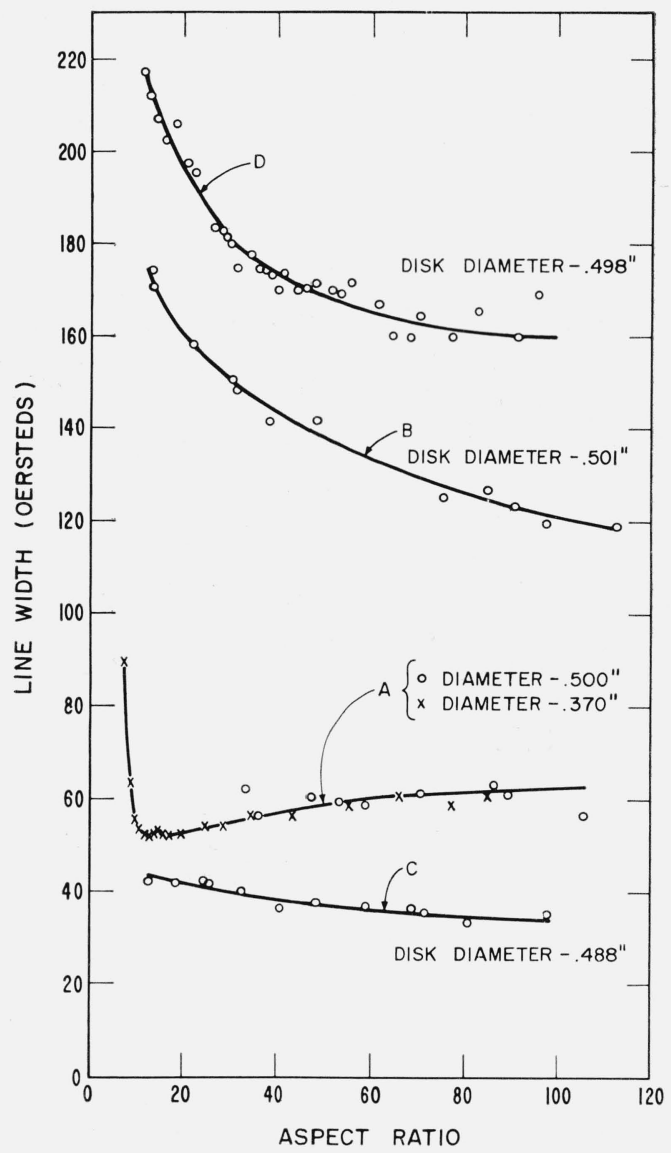

FIGURE 4. Line width versus aspect ratio for disk samples of materials $A, B, C$, and $D$.

\subsection{Line Width}

Figure 4 shows the line width of materials A through $D$ as a function of the aspect ratio of the disk-shaped samples. Here again, it is seen that it is difficult to obtain disks sufficiently thin in order to obtain an asymptotic value of line width. It may thus be necessary to obtain data at several different values of aspect ratio and extrapolate the results to the higher aspect ratios in order to obtain meaningful information on line width. It is readily apparent that samples B, C, and D are displaying similar phenomena in that they show a relatively slow decrease in line width as the aspect ratio increases. This decrease in line width with increasing aspect ratio is probably due to inhomogeneous field effects as well as incomplete saturation of the individual crystallites. The inhomogeneous field effect might be expected to result in magnetostatic modes arising from field inhomogeneities due to spacial variations in the demagnetizing field resulting from deviation of the disks from true ellipsoids such as described by Geschwind and Clogston [10]. The problem of incomplete saturation might be expected to cause a line broadening if the relatively low internal d-c fields in the disk required for resonance at L-band become of the same order of magnitude as the anisotropy fields such as discussed by Suhl et al. [11]. The greatest variation in line width with aspect ratio in the data given in figure 4 appears on the low field side of the resonance curves. This effect might be expected for both line broadening mechanisms described above. As such, it does not appear possible at the present time to separate the effects due to saturation from the various possible inhomogeneous field effects that may be present in this data. One further point of interest in regard to this data is that sample D does not display an anomalous behavior in line width even though there was a strong dependence of resonant field on diameter and surface finish.

Sample $\dot{A}$, which is yttrium iron garnet, shows a somewhat different dependence of line width with aspect ratio. For this case, the line width appears to be relatively constant for the higher aspect ratios. As can be seen, it is possible that there is actually a slight increase in line width as the aspect ratio 
increases in this material. This apparent increase in line width for the higher aspect ratios is not entirely understood although here again, the increase is largely due to changes in the low field side of the resonance curve. It is also of interest to note that the resonance curves for this material were in general more symmetrical than for the previous materials B, C, and D. This is in agreement with data obtained on single crystal disks of YIG by Smith and Watanabe [12]. The $1 / 2$ in. diam samples of this material proved to be too lossy for measurements at the lower aspect ratios. However, data taken on a smaller $3 / 8$ in. diam sample of yttrium iron garnet indicated that there is a very large increase in line width at aspect ratios of the order of ten or less. This is presumably due to the fact that these samples are becoming magnetically unsaturated at the lower aspect ratios. It should also be noted that variations in data on different samples of the same diameter cut from the same block were noted for this material. This apparent inhomogeneity in such materials considerably effects the precision of measurements such as described above when different samples must be used.

A final remark concerning the comparison of line width measurements between ellipsoids and disks of the same aspect ratio for the above four materials may be of interest. It has previously been mentioned that a negligible variation in resonant field was noted when a disk was ground to an ellipsoid of the same aspect ratio. However, for the case of samples B, C, and D, the ellipsoid had a smaller line width than the disk of the same aspect ratio. For sample $A$, the ellipsoid had a greater line width than the corresponding disk. This suggests that magnetostatic mode phenomena affects the line width but not the resonant field in these polycrystalline materials.

\section{Conclusions}

1. The use of a $\mathrm{TE}_{102}$ rectangular cavity is quite applicable for measuring the effective line width and gyromagnetic ratio of disk-shaped samples at L-band frequencies.

2. In many cases, it may be necessary to obtain line width and gyromagnetic ratio as a function of aspect ratio and extrapolate the data to obtain the asymptotic values for these quantities. However, for many materials, the gyromagnetic ratio of a finite disk may be calculated from Kittel's equation if one uses the appropriate demagnetizing factors and has a value of $M$ available.

3 . In at least one case such as nickel aluminum ferrite, it is not possible to fit Kittel's equation in its usual form to the curves giving resonant field as a function of aspect ratio. Such an anomaly appears to be associated with both a diameter and surface finish dependence of $H_{r}$ as a function of the aspect ratio. Considerable care should thus be given to the interpretation of data of this type on such materials at L-band frequencies.

\section{References}

[1] Artman, J. O., and P. E. Tannenwald, Measurement of susceptibility tensor in ferrites, J. Appl. Phys. 26, No. 9, 1124-1132 (1955).

[2] Soohoo, R. F., Theory and application of ferrites, pp. 92-99 (Prentice-Hall, Inc., Englewood Cliffs, New Jersey, 1960).

[3] Sirvetz, M. H., and J. E. Zneimer, Microwave properties of polycrystalline rare earth garnets, J. Appl. Phys. 29, 431 (1958).

[4] Lax, B., and K. J. Button, Microwave ferrites and ferrimagnetics, pp. 484-485 (McGraw-Hill Company, Inc., New York, N.Y., 1962).

[5] Kittel, C., On the theory of ferromagnetic resonance aksorption, Phys. Rev. 73, 155-161 (1948).

[6] Preston, C. C., and W. E., Case, Tables to facilitate the determination of the ferrimagnetic resonance line width of non-metallic magnetic materials, NBS Tech. Note No. 173 (Apr. 1963).

[7] Von Aulock, W., and J. H. Rowen, Measurement of dielectric and magnetic properties of ferromagnetic materials at microwave frequencies, Bell System Tech. J. XXXVI, No. 2, 427 (1947).

[8] Stoner, E. C., The demagnetizing factors for ellipsoids, Phil. Mag. (7) 36, 803-821 (1945).

[9] Dillon, J. F., Magnetostatic modes in disks and rods, J. Appl. Phys. 31, No. 9, 1605-1614 (Sept. 1960).

[10] Geschwind, S., and A. M. Clogston, Narrowing effect of dipole forces on inhomogeneously broadened lines, Phys. Rev. 108, No. 1, 49-53 (Oct. 1957).

[11] Suhl, H., L. G. Van Uitert, and J. L. Davis, Ferromagnetic resonance in magnesium-manganese aluminum ferrite between 160 and 1900 Mc, J. Appl. Phys. 26, No. 9, 1180 (Sept. 1955).

[12] Smith, A. W., and A. Watanabe, Ferrimagnetic resonance in a single-crystal disk of yttrium iron garnet, J. Appl. Phys., Supplement to Volume 32, No. 3, $155 \mathrm{~S}$ (1961).

(Paper 68C2-154) 